\title{
ORIGINAL
}

\section{RELACIÓN DEL CONSUMO DE ALCOHOL Y DROGAS DE LOS JÓVENES ESPAÑOLES CON LA SINIESTRALIDAD VIAL DURANTE LA VIDA RECREATIVA NOCTURNA EN TRES COMUNIDADES AUTÓNOMAS EN 2007 (*)}

\author{
Amador Calafat Far (1), Daniel Adrover Roig (2), Montserrat Juan Jerez (1), Nicole T. Blay \\ Franzke (1)
}

(1) IREFREA, Instituto y Red Europea para el Estudio de los Factores de Riesgo en la Infancia y la Adolescencia. Palma de Mallorca.

(2) UIB Universitat de les Illes Balears, Facultad de Psicología. Palma de Mallorca.

\section{RESUMEN}

Fundamento: El fenómeno conocido como consumo concentrado de alcohol y otras drogas se produce de manera frecuente entre los jóvenes en las áreas recreativas nocturnas. El presente estudio analiza las conductas de riesgo y la siniestralidad asociada al alcohol y otras drogas en una muestra de jóvenes que participan de la vida recreativa nocturna.

Método: Participaron 440 jóvenes de 3 comunidades autónomas. El tamaño de la muestra fue estimado mediante Respondent Driven Sampling. Los análisis estadísticos exploraron frecuencias y medidas de asociación de conductas de riesgo en conducción en relación con la siniestralidad, número de estados de embriaguez, frecuencia de accidentes en el último mes, influencia de sexo y edad, así como la potencia predictiva de dichas variables respecto a la siniestralidad.

Resultados: Un 50,2\% de los jóvenes ha subido con un conductor ebrio y/o drogado, un $23,2 \%$ ha conducido embriagado y un $23,5 \%$ bajos los efectos de otras drogas. A mayor implicación en la noche, mayor frecuencia de embriaguez $\left[\operatorname{chi}^{2}(9)=112,24 ; \mathrm{p}<, 000\right]$; a mayor número de borracheras, mayor frecuencia en las conductas: subirse con otros conductores ebrios o drogados $\left[\right.$ chi $^{2}=36.442$, , (3) $\mathrm{p}<0.001]$, conducir ebrio $\left[\mathrm{chi}^{2}=23,748,(3) \mathrm{p}<0,001\right]$ y conducir drogado $\left[\right.$ chi $\left.^{2}=23,816(3) \mathrm{p}<0,001\right]$. El análisis de regresión destacó conducir ebrio (odds-ratio $=5,4$ ) como conducta de riesgo más asociada a los accidentes de tráfico.

Conclusiones: Las conductas de riesgo durante la conducción de vehículos muestran una elevada incidencia. Embriaguez, uso de drogas e implicación en la noche aumentan la frecuencia de dichas conductas. Conducir ebrio fue el mejor predictor de los accidentes.

Palabras clave: Trafico, accidente. Prevención, accidente. Jóvenes. Alcohol. Drogas ilegales.

Correspondencia:

Nicole T. Blay Franzke

IREFREA

C/ Ramblas, $15,2^{\circ}, 3^{\text {a }}$

07002 Palma de Mallorca.

nicole@irefrea.org
ABSTRACT

Relationship between Alcohol, Drug Use and Traffic Accidents Related to Nightlife among a Spanish Youth Sample in Three Autonomous Communities in 2007

Background: The phenomenon known as binge drinking and other drug abuse frequently occurs among young people in nightliferelated areas. This study analyzes the risk behaviours and the accident rate related to alcohol and other drugs among a sample of young people involved in nightlife.

Method: A total of 440 young people from 3 Spanish Autonomous Communities took part. The sample size was estimated by means of Respondent Driven Sampling. The statistical analyses mined frequencies and measurements of the relationship of driving risk-related behaviour in relation to the accident rate, number of cases of drunkenness, frequency of accidents during the past month, influence of gender and age, as well as the predictiveness of these variables as regards the accident rate.

Results: A total of $50.2 \%$ of these young people had gotten into a vehicle with a driver who was drunk and/or under the influence of drugs, $23.2 \%$ has driven when drunk and $23.5 \%$ under the influence of drugs. The greater the degree of involvement in nightlife, the higher the frequency of drunkenness $\left[\mathrm{chi}^{2}(9)=112.24\right.$; $\left.\mathrm{p}<.000\right]$; the greater the number of cases of drunkenness, the higher the frequency of thes behaviors: getting into a vehicle with drivers who are drunk or under the influence of drugs $\left[\mathrm{c}^{2}=36,442,(3) \mathrm{p}<0.001\right]$, drunken driving $\left[\mathrm{chi}^{2}=23,748,(3)\right.$ $\mathrm{p}<0.001]$ and driving while under the influence of drugs $\left[\mathrm{chi}^{2}=23,816\right.$ (3) $\mathrm{p}<0.001]$. The regression analysis highlighted drunken driving (odds-ratio $=5.4$ ) as the risk behaviour most related to traffic accidents.

Conclusions: There is a high incidence of risk behaviours while driving vehicles. Drunkenness, drug use and involvement in nightlife increase the frequency of these behaviours. Drunken driving was the best accident predictor.

Keywords: Accidents, traffic. Accident Prevention. Youth. Alcohol. Illicit drugs. Drinking.

(*) Este estudio se ha realizado en parte con financiación de la Delegación del Gobierno para el Plan Nacional sobre Drogas según la Orden de convocatoria SCO/2646/2007 y con financiación del Health and Consumer Protection Directorate General (European Commission; grant agreement 2004319). 


\section{INTRODUCCIÓN}

Se estima que cada año mueren en el mundo 1,2 millones de personas a causa de accidentes en la vía pública y otros 50 millones resultan heridas. Sin un renovado compromiso con la prevención, las proyecciones indican que estas cifras aumentarán en torno al $65 \%$ en los próximos 20 años ${ }^{1}$. Se calcula que una de cada cuatro muertes en carretera entre varones de 15 a 29 años es atribuible al abuso de alcohol ${ }^{2}$. Está bien establecido que el alcohol es un factor principal en las heridas y muertes no sólo de conductores ebrios sino de otros usuarios de las vías ${ }^{3}$. Según autoinforman los jóvenes universitarios de un estudio español, conducir después de consumir alcohol es una práctica generalizada entre ellos y en algunos casos incluso después de haber consumido dosis elevadas ${ }^{4}$.

Por otra parte, un $35 \%$ de los consumidores habituales de cannabis reconoce que con frecuencia se pone al volante en las cuatro horas siguientes al consumo de la $\operatorname{droga}^{5}$. Los efectos negativos del cannabis sobre la conducción han sido ampliamente documentados $^{6-9}$, así como también los diversos efectos posibles del consumo combinado de alcohol y cannabis sobre la conducción ${ }^{3,10}$, quedando su influencia fuera de toda duda.

Existe amplia literatura congruente sobre las diferencias de género en conducción de riesgo según la cual son los varones jóvenes los que con mayor frecuencia se ven involucrados en los accidentes con heridos ${ }^{11}$. Los varones informan asumir mayor número de conductas de riesgo en conducción ${ }^{12,4}$ mientras que las mujeres muestran mayor grado de cumplimiento de la normativa de tráfico ${ }^{12}$ y una actitud más positiva hacia la seguridad vial ${ }^{13}$.

También existe evidencia de que la vida recreativa nocturna es un factor de riesgo para el consumo de alcohol y otras drogas por parte de los jóvenes ${ }^{14,15}$ y también para otras conductas de riesgo ${ }^{16}$. El fenómeno conocido como binge-drinking (consumo concentrado de alcohol de una sentada) y otras drogas se produce de manera frecuente entre los jóvenes en las áreas recreativas nocturnas ${ }^{17,18}$. La presencia de conductas de riesgo durante la conducción es elevada entre los jóvenes en el contexto recreativo $^{19,20}$.

En los estudios tradicionales sobre conducción se consideraba como principal conducta de riesgo conducir ebrio y conducir drogado. Sin embargo, varios estudios han manifestado la importancia de la conducta de riesgo de subirse a un vehículo cuyo conductor está ebrio y/o drogado ${ }^{21}$. Un estudio europeo muy reciente muestra además la elevada prevalencia de esta conducta entre los jóvenes (datos sin publicar).

Este estudio tiene como objetivos explorar entre los jóvenes que suelen salir de marcha los fines de semana la incidencia de las conductas de riesgo en conducción (subirse con un conductor ebrio o drogado, conducir ebrio, conducir drogado), la incidencia de los accidentes debidos a consumos de alcohol y otras drogas, y la relación que se establece con diversos factores de riesgo: sexo, consumos y el grado de implicación en la vida recreativa nocturna.

\section{SUJETOS Y MÉTODO}

La muestra la formaron 440 jóvenes de entre 14-25 años [media (d.e): 21.14 (2,76); $47,7 \%$ varones] que frecuentan la actividad recreativa nocturna, y son consumidores de alguna droga legal o ilegal, entrevistados entre los meses de abril a julio de 2007 en tres comunidades españolas (Baleares, Galicia y Valencia). Todos los sujetos participaron de forma voluntaria, bajo la garantía del anonimato, y respetándose todos los acuerdos de la Declaración de Helsinki en su revisión del año 2000 durante la investi- 
gación. Para la selección de la muestra se ha utilizado el método Respondent Driven Sampling (RDS). Este método de muestreo fue diseñado especialmente para el estudio de poblaciones carentes de marco muestral y de difícil acceso $^{22}$. La aplicación del RDS se inicia con la identificación de los informantes iniciales que cumplen la función de "semillas". A partir de la muestra real obtenida se crea matemáticamente una muestra en equilibrio que después se compara con la muestra real. Nosotros utilizamos las variables "sexo" y "grupos de edad" para elaborar las muestras en equilibrio. Con la primera obtuvimos una diferencia media entre los valores de la muestra real y la muestra en equilibrio del $0,4 \%$. En el caso de la variable "grupos de edad" esta diferencia fue del $1,3 \%$. Estos índices señalan que nos movemos dentro de una tolerancia menor del $2 \%$ por lo que hemos logrado una aproximación muy aceptable a la muestra matemática teóricamente en equilibrio. Para poder aplicar este método ha resultado imprescindible el uso del software específico RDSAT (Respondent Driven Sampling Analysis Tool v. 5.0.1.).

Para la creación de una escala que permitiera valorar la participación en la vida recreativa nocturna de los sujetos se creó un índice a partir de la integración de los siguientes ítems: veces que han salido de marcha en las últimas cuatro semanas; noches del fin de semana que han salido de marcha + cuando salen de marcha, horas que suelen estar + número de sitios a los que van cuando salen de marcha. El resultado fue la creación de una variable continua que define el grado la implicación en la noche (de $<=1$, a $>=4$ ).

Los análisis estadísticos exploraron las frecuencias y medidas de asociación de conductas de riesgo en conducción en relación con la siniestralidad, el número de borracheras y la frecuencia de accidentes en el último mes. La influencia de los factores sexo y edad, tanto en las conductas de ries- go como en la frecuencia del consumo de alcohol y drogas y siniestralidad, fue explorada mediante el estadístico ji-cuadrado. Para tratar de estimar la capacidad predictiva tanto de los consumos de alcohol y drogas durante la noche como de los diferentes factores de riesgo asociados a la conducción, se llevaron a cabo análisis multivariantes con métodos de regresión logística. La variable criterio de tipo dicotómico a predecir fue el haber tenido un accidente de tráfico como consecuencia del consumo del alcohol u otras drogas en los últimos 30 días. En un primer paso de la regresión se introdujeron las variables referentes tanto al consumo (borracheras y consumos de diferentes tipos de drogas ilegales) como a las conductas de riesgo asociadas a la conducción que se habían relacionado significativamente con los accidentes de tráfico. En el segundo paso de la regresión se introdujeron aquellos predictores que en el paso anterior habían mostrado ser significativos en el modelo. Análisis de regresión logística para predecir la siniestralidad: predictores para dos tipos de variables: Coeficientes: odds-ratio, confianza al $95 \%$ y valor de significación de cada predictor de la ecuación de regresión logística. Primer paso de la regresión: predictores en cada tipo de variables: odds ratio (intervalo de confianza al 95\%, significación. Segundo paso de la regresión: mejores predictores seleccionados tras el primer paso en cada tipo de variables: odds ratio (intervalo de confianza al 95\%, significación)

Dichos análisis fueron llevados a cabo mediante el paquete estadístico SPSS v. 12.0.

\section{RESULTADOS}

\section{Conductas de riesgo durante la conducción}

El 50,2\% de los jóvenes informa haberse subido alguna vez a un vehículo conducido 
por un conductor ebrio o bajo la influencia de otras drogas (últimos 30 días). El 23,2\% informa haber conducido embriagado él mismo, y un $23,5 \%$ estando bajo el efecto de alguna droga.

Respecto al sexo observamos que no existen diferencias estadísticamente significativas entre los hombres y las mujeres para la conducta de riesgo subirse al vehículo con otro conductor ebrio o drogado $\left[\chi^{2}(17)=27,34 \mathrm{p}=0,066\right]$, pero sí para la conducta de conducir ebrio $\left[\chi^{2},(12)=33,73\right.$ $\mathrm{p}<0,001$ ] y conducir estando bajo los efectos de alguna droga $\left[\chi^{2}(15)=46,72\right.$; $\mathrm{p}<, 0001]$, siendo los varones los que presentan con mayor frecuencia estas dos conductas.

No se hallaron diferencias estadísticamente significativas en función de la edad para ninguna de las conductas de riesgo en conducción: subirse al vehículo con otros conductores ebrios o drogados $\left[\chi^{2}\right.$ $(17)=22,02 \mathrm{p}=0,84]$, conducir bajo los efectos de alguna droga $\left[\chi^{2}(15)=13,54 \mathrm{p}=0,56\right]$ ni para la conducta de conducir ebrio $\left[\chi^{2}\right.$ $(1)=1,63 \mathrm{p}=0,201]$. Las tres conductas de riesgo en conducción presentan una correlación directa y significativa: la conducta "subirse con otros conductores ebrios o drogados" se relacionó significativamente con "conducir ebrio" [r=0,295; $\mathrm{p}<0,001] \mathrm{y}$ con la conducta de riesgo "conducir droga$d o$ " $[\mathrm{r}=0,212 \mathrm{p}<0,001]$. Asimismo, la conducta de riesgo conducir ebrio se correlacionó significativamente con conducir drogado $[\mathrm{r}=0,532 \mathrm{p}<0,001]$. La correlación más significativa, por tanto, se dió entre las conductas de conducir ebrio y la de conducir drogado [r=0,532 $\mathrm{p}<0,001]$.

\section{Borracheras y consumo de drogas ilegales}

Respecto a la conducta de emborracharse el $19,4 \%$ refirió no haberlo hecho, un $16,4 \%$ haberlo hecho una vez, un $19,2 \%$ dos veces y un $45 \%$ se había emborrachado más de dos veces. No hubo diferencias estadísticamente significativas por grupos de edad $\left[\chi^{2}(3)=3,28 ; p=0,35\right]$, aunque sí por género $\left[\chi^{2}(3)=21,14 ; p<0,0001\right]$, de manera que en términos generales los varones presentaron mayor número de borracheras (88\% al menos una vez al mes) que las mujeres (73,5\% al menos una vez al mes). Sin embargo, fueron las mujeres $(18,6 \%)$, en comparación con los varones $(13,9 \%)$, las que con mayor frecuencia se emborrachan una vez al mes.

La droga ilegal consumida con mayor frecuencia fue el cannabis: el $15 \%$ refirió no haberlo probado nunca, un $12,5 \%$ lo consumía de manera ocasional (menos de una vez al mes hasta una vez a la semana) y un $54,3 \%$ lo hacía frecuentemente (más de una vez a la semana). No se apreciaron diferencias significativas en cuanto a la edad $\left[\chi^{2}(7)=10,7 ; \mathrm{p}=0,15\right]$, aunque sí por sexo $\left[\chi^{2}(7)=20,12 ; p<0,005\right]$, de tal manera que había casi el doble de mujeres no consumidoras, ex-consumidoras o que lo habían probado 1 ó 2 veces $(64,1 \%$ frente a $35,9 \%$ varones). Hubo mayor porcentaje de varones consumidores ocasionales y frecuentes $(53,8 \%$ varones vs. $46,2 \%$ mujeres en ambos casos).

La segunda droga ilegal consumida con mayor frecuencia fue la cocaína. Un 47,9\% no lo había probado nunca frente a un $19,6 \%$ que refirió consumirla de manera ocasional (menos de $1 \mathrm{vez}$ al mes hasta 3 veces a mes), y un $10,1 \%$ de manera frecuente (1 vez a la semana o más). Hubo diferencias estadísticamente significativas en función de la edad $\left[\chi^{2} \quad(7)=20,63\right.$ $\mathrm{p}=0,004]$, siendo el grupo de 19-25 años el que presenta mayores frecuencias de consumo, y del sexo $\left[\chi^{2}(7)=30,64 ; p=0,0001\right]$, siendo los varones los que mayores frecuencias de consumo presentaron.

Respecto al éxtasis, un $65,3 \%$ no lo había probado nunca, un $12,5 \%$ lo había probado una o dos veces, y un $6,6 \%$ eran 
ex-consumidores de esta droga. Un 13,3\% consumía de manera ocasional (menos de 1 vez al mes hasta 3 veces a mes), y un 2,4\% lo hacía de manera frecuente (1 vez a la semana o más). Hubo diferencias significativas tanto por sexo $\left[\chi^{2}(6)=13,03\right.$; $\mathrm{p}=0,043]$, como por edad: $\left[\chi^{2}(6)=17,32\right.$; $\mathrm{p}=0,008]$, siendo mayor la frecuencia de consumo para el grupo de los mayores de 18 años.

\section{Participación en la noche en relación a borracheras y consumo de drogas ilegales}

A medida que aumenta la implicación en la vida recreativa nocturna se reduce el porcentaje de personas que refirió no haberse emborrachado en las últimas cuatro semanas, y aumentó el de los que se emborracharon con frecuencia (más de 2 veces al mes), es decir, a mayor participación en la noche mayor frecuencia de borracheras $\left[\chi^{2}(9)=\right.$ $112,24 ; \mathrm{p}<0,000]$.

Sucedió lo mismo respecto al consumo de cannabis $\left[\chi^{2}(21)=62,15 ; \mathrm{p}<0,000\right] \mathrm{de}$ cocaína $\left[\chi^{2}(21)=54,76 ; p<0,000\right]$ y de éxtasis $\left[\chi^{2}(18)=52,1 ; \mathrm{p}=<000\right]$, por tanto, a mayor participación en la noche mayor porcentaje de personas que consumen drogas, en especial en las categorías de mayor consumo.

\section{Relación de conductas de riesgo durante la conducción con consumos y participación en la vida nocturna}

Se hallaron diferencias estadísticamente significativas entre borracheras y diferentes conductas de riesgo, de tal forma que a mayor número de borracheras mayor frecuencia en dichas conductas: subirse con otros conductores ebrios o drogados $\left[\chi^{2}=36,442\right.$, (3) $\left.\mathrm{p}<0,001\right]$, conducir ebrio $\left[\chi^{2}=23,748\right.$, (3) $\left.\mathrm{p}<0,001\right]$ y conducir drogado $\left[\chi^{2}=23,816\right.$ (3) $\left.\mathrm{p}<0,001\right]$. Del mismo modo, se hallaron diferencias estadísticamente significativas entre las tres conductas de riesgo y la frecuencia de consumo de cannabis, cocaina y éxtasis, con un patrón similar al de las borracheras (tabla 1).

Tabla 1

Borracheras, consumo de drogas ilegales y conductas de riesgo en conducción

\begin{tabular}{|c|c|c|c|}
\hline & $\begin{array}{c}\text { Subir a un vehículo } \\
\text { conducido por alguien } \\
\text { bebido o bajo la influencia } \\
\text { de sustancias }\end{array}$ & $\begin{array}{l}\text { Conducir un vehículo } \\
\text { tras haber bebido }\end{array}$ & $\begin{array}{c}\text { Conducir un vehículo } \\
\text { bajo el efecto de sustancias }\end{array}$ \\
\hline $\begin{array}{l}\text { Alcohol (borracheras) } \\
\text { Nunca } \\
1 \text { vez } \\
2 \text { veces } \\
>2 \text { veces }\end{array}$ & $\begin{array}{c}\chi^{2}=36,442 * * * \\
28,2 \% \\
35,2 \% \\
52,4 \% \\
63,4 \%\end{array}$ & $\begin{array}{c}\chi^{2}=23,748 * * * \\
5,9 \% \\
18,3 \% \\
22 \% \\
31,8 \%\end{array}$ & $\begin{array}{c}\chi^{2}=23,816^{* * * *} \\
9,4 \% \\
11,3 \% \\
28 \% \\
31,8 \%\end{array}$ \\
\hline $\begin{array}{l}\text { Cannabis } \\
\text { Nunca } \\
\text { Ex consumidor } \\
\text { Ocasional } \\
\text { Frecuente } \\
\end{array}$ & $\begin{array}{c}\chi^{2}=15,057 * * \\
38,8 \% \\
48,8 \% \\
42,2 \% \\
59,9 \%\end{array}$ & $\begin{array}{c}\chi^{2}=11,031 * \\
12,6 \% \\
19,5 \% \\
22,2 \% \\
29,3 \%\end{array}$ & $\begin{array}{c}\chi^{2}=64,665^{* * * *} \\
4,9 \% \\
7,3 \% \\
14,4 \% \\
41,4 \%\end{array}$ \\
\hline $\begin{array}{l}\text { Cocaina } \\
\text { Nunca } \\
\text { Ex consumidor } \\
\text { Ocasional } \\
\text { Frecuente }\end{array}$ & $\begin{array}{c}\chi^{2}=20,947 * * * \\
41,6 \% \\
53,6 \% \\
68,4 \% \\
64,1 \%\end{array}$ & $\begin{array}{c}\chi^{2}=25,964 * * * \\
17,1 \% \\
25,0 \% \\
36,7 \% \\
48,7 \%\end{array}$ & $\begin{array}{c}\chi^{2}=55,648 * * * \\
14,7 \% \\
17,9 \% \\
39,2 \% \\
64,1 \%\end{array}$ \\
\hline $\begin{array}{l}\text { Éxtasis } \\
\text { Nunca } \\
\text { Ex consumidor } \\
\text { Ocasional } \\
\text { Frecuente }\end{array}$ & $\begin{array}{c}\chi^{2}=9,484 * \\
45,2 \% \\
65,4 \% \\
59,2 \% \\
77,8 \%\end{array}$ & $\begin{array}{c}\chi^{2}=14,521 * * \\
19,9 \% \\
38,5 \% \\
36,0 \% \\
55,6 \%\end{array}$ & $\begin{array}{c}\chi^{2}=13,974 * * \\
19,5 \% \\
38,5 \% \\
34,0 \% \\
55,6 \%\end{array}$ \\
\hline
\end{tabular}

$* \mathrm{p}<0,05 * * \mathrm{p}<0,01 * * * \mathrm{p}<0,001$. 
Por otra parte, a mayor grado de participación en la noche, mayor frecuencia de las conductas de riesgo en conducción subirse con otros conductores ebrios o drogados $\left[\chi^{2}=11,424,(3) \mathrm{p}<0,01\right]$ y conducir drogado $\left[\chi^{2}=15,606\right.$ (3) $\left.\mathrm{p}<0,01\right]$. No se hallaron diferencias para la conducta de riesgo conducir ebrio en función de la participación en la vida nocturna.

\section{Conductas de riesgo y accidentes}

El 5,3\% de las personas de la muestra informó haber sufrido un accidente de tráfico durante los últimos 12 meses a consecuencia de su consumo de alcohol y/o otras drogas.

A pesar de que la conducta de riesgo durante la conducción esmás frecuente $(50,2 \%)$ en términos absolutos es la de subirse con otro conductor ebrio o droga$d o$, cuando atendemos específicamente a la submuestra de los que informan haber sufrido accidentes, observamos que la con- ducta de riesgo que se asocia con mayor frecuencia $(65,2 \%)$ es la de conducir uno mismo ebrio seguida de la de conducir drogado. La conducta de riesgo que aparece con menor frecuencia $(4,3 \%)$ asociada a la siniestralidad es la de subirse con otros conductores ebrios o drogados (figura 1).

\section{Predictores de los accidentes}

Dado que tanto consumos de drogas como las frecuencias en las conductas de riesgo, y el sexo y edad fuero variables que se asociaron de forma significativa con la siniestralidad, se efectuó un análisis de regresión logística (variable criterio: siniestralidad) con el objeto de identificar aquellos factores que de forma más directa influyen en la siniestralidad. El análisis de regresión logística respecto de las variables asociadas al consumo, incluyó 4 variables con un buen ajuste $\left(\chi^{2} \mathrm{p}<0,0001\right)$. Por otra parte, el análisis de regresión logística con los factores asociados al riesgo en conducción consistió en 3 variables con un ajuste:

Figura 1

Coductas de riesgo asociadas a la siniestralidad

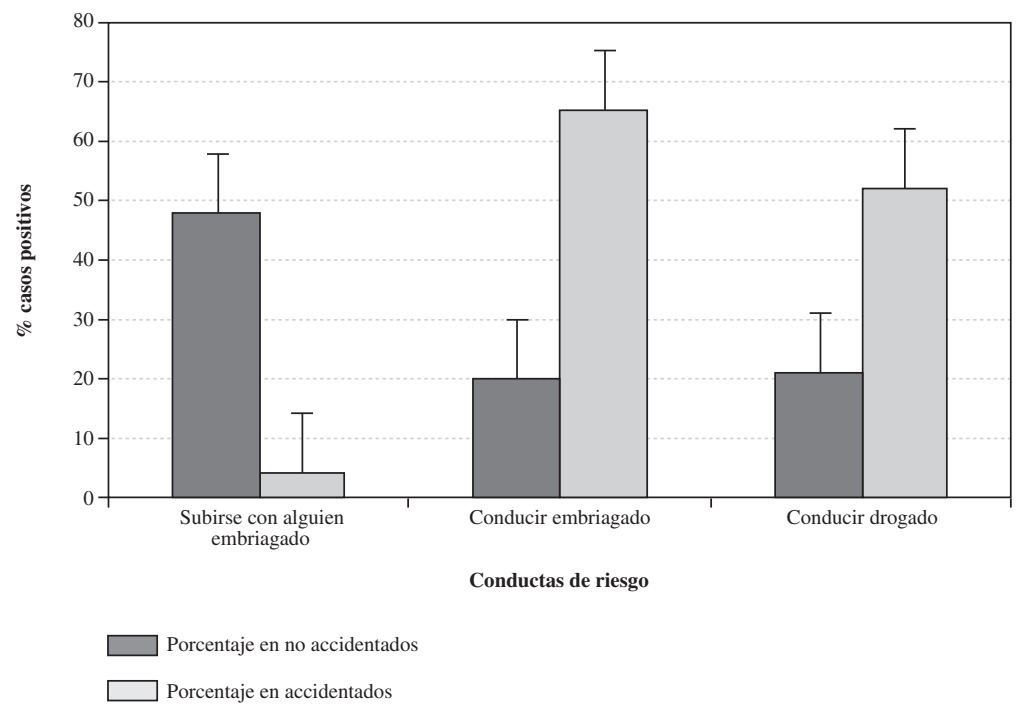


Predictores de los accidentes. Resultados de la regresión

\begin{tabular}{|c|c|c|}
\hline Variable & Primer paso de regresión & Segundo paso de regresión \\
\hline $\begin{array}{l}\text { Factores relacionados con el consumo de alcohol y drogas } \\
\text { Número de borracheras* } \\
\text { Consumo de cannabis } \\
\text { Consumo de cocaína } \\
\text { Consumo de éxtasis }\end{array}$ & $\begin{array}{l}1,81(1,1-3,2) 0,043 * \\
0,90(, 59-1,37) 0,618 \\
1,36(0,83-2,2) 0,212 \\
1,50(0,87-2,57) 0,140\end{array}$ & $1,76(1-3,1) 0,047^{*}$ \\
\hline $\begin{array}{l}\text { Factores de riesgo en conducción } \\
\text { Subirse con un conductor ebrio o drogado } \\
\text { Conducir ebrio** } \\
\text { Conducir drogado }\end{array}$ & $\begin{array}{l}2,7(0,84-8,64) ; 0,094 \\
5,17(1,68-15,8) 0,004 * * \\
1,50(0,52-4,27) 0,45\end{array}$ & $5,43(2,16-13,64) ; 0,000 * * *$ \\
\hline $\begin{array}{l}\text { Variables sociodemográficas relacionadas } \\
\text { Edad } \\
\text { Sexo }\end{array}$ & $\begin{array}{c}0,93(0,79-1,1) \\
1,41 \\
1,41(0,9-1,33) 0,007 * *\end{array}$ & $0,626(0,36-1,23) ; 0,115$ \\
\hline
\end{tabular}

$* \mathrm{p}<0,05 * * \mathrm{p}<0,01 * * * \mathrm{p}<0,001$.

$\left(\chi^{2} \mathrm{p}<0,0001\right)$. El análisis de regresión logística con los factores sociodemográficos (edad y sexo) también contó con un buen ajuste $\left(\chi^{2} p<0,01\right)$. En el primero de los dominios, la variable número de borracheras se perfiló como un predictor de los accidentes, en el segundo el mejor de los predictores fue la conducta de riesgo de conducir ebrio, y en el tercero s fue la variable género la que se perfiló como mejor predictora (tabla 2). Dado que los mejores predictores de los accidentes fueron 3 (el número de borracheras en el dominio consumo y el conducir ebrio en el dominio riesgos y el ser hombre), la ecuación definitiva consistió en 3 variables capaces de clasificar correctamente el 96,7 $\%$ de los sujetos del total de la muestra. Cabe destacar que el más potente de los predictores fue la conducta de riesgo conducir ebrio (tabla 2), seguido del número de borracheras. En el segundo paso de la regresión la variable sexo perdió su significación estadística, muy probablemente debido a la variancia compartida de esta variable con el número de borracheras y la conducta de riesgo de conducir ebrio.

\section{DISCUSIÓN}

Según los resultados de este estudio son los jóvenes varones los que presentan mayores frecuencias de consumos de alcohol y otras drogas y también de conductas de riesgo, al igual que en otro estudio ${ }^{13}$, por lo que se trata de un grupo de riesgo para el que cabría diseñar actuaciones preventivas específicas. Sin embargo, no debemos descuidar el hecho de que las mujeres se emborrachan con mayor frecuencia de manera ocasional (una o dos veces al mes). Este dato, junto con la evidencia demostrada recientemente de que conductores con borracheras ocasionales están en mayor riesgo de sufrir accidentes (datos sin publicar) nos pone sobre aviso de que las mujeres jóvenes también son un colectivo de alto riesgo.

Nuestros datos muestran que a mayor grado de participación en la vida nocturna mayor frecuencia de borracheras y de consumos de otras drogas ilegales, lo cual coincide con otros estudios que apuntan al ámbito recreativo nocturno como facilitador de estos consumos ${ }^{14,15,17}$. De la misma forma, a mayor participación en la vida nocturna mayor frecuencia de conductas de riesgo relacionadas con la conducción, al igual que a mayor consumo mayor frecuencia de conductas de riesgo, por lo que cabe suponer que todas ellas son variables relacionadas. Cabe por ello pensar que todas aquellas medidas pensadas tanto para disminuir la participación en la vida nocturna (a través 
de controlar los horarios, aumentar precios, control por los padres de los horarios de salida, etc.) como limitar los consumos, pueden tener efectos sobre la siniestralidad asociada al alcohol y otras drogas ${ }^{23}$.

A pesar de que los datos muestran que a mayor participación en la vida nocturna mayor frecuencia de las conductas de riesgo "subirse con otras conductores ebrios" y "conducir uno mismo drogado", esto no sucede con la conducta "conducir uno mismo ebrio". Este dato, difícil de interpretar, podría hallar explicación en el hecho de que en ocasiones las personas que más beben están posiblemente adoptando más conductas de reducción del daño, es decir, utilizan menos el coche privado. Una situación similar se encontró también en un estudio con población de iguales características, en el que los que más se emborrachaban cogían menos el coche privado (datos sin publicar).

En relación con la importancia del sexo conducir ebrio o drogado son conductas más frecuentes en los varones, sin embargo, precisamente la conducta de riesgo que se produce con más frecuencia, subirse con otro conductor ebrio o drogado, se asume de manera similar por varones y mujeres. Seguramente no existe suficiente conciencia social de la peligrosidad de esta conducta de riesgo, por lo que las medidas preventivas deberían ir dirigidas a sensibilizar sobre este problema. Cabe la posibilidad de que las mujeres sean especialmente sensibles a estas campañas, pues han demostrado más prudencia con otras conductas de riesgo, como el consumo de drogas. Por otro lado en otros estudios se demuestra que suelen ser más los varones los que conducen su coche privado cuando acuden a los lugares recreativos nocturnos (datos sin publicar).

Las tres conductas de riesgo en conducción exploradas en el estudio presentan una correlación positiva y significativa entre sí. Por tanto, las intervenciones preventivas en conducción segura dirigida a los jóvenes deberían abarcar todas las posibles conductas de riesgo, y cómo el joven puede hacerles frente y desarrollar estrategias adecuadas para evitarlas.

En resumen, según se desprende de los resultados de los análisis de regresión logística, aunque la frecuencia de borracheras y el hecho de ser varón se perfilan como predictores de los accidentes de tráfico, la conducta de conducir ebrio cuenta con la mayor potencia predictiva. Esta conducta, como es lógico, presupone el hecho de embriagarse con anterioridad y se produce de forma más frecuente en varones que en mujeres.

El presente estudio presenta ciertas limitaciones sobre el poder de extrapolación y generalización de los resultados. Al ser la muestra seleccionada según los criterios de inclusión participación en la vida recreativa nocturna y consumo de una o varias drogas legales y/o ilegales, implica que todos los resultados se pueden referir sólo a los patrones de comportamiento de esta muestra. También hay que considerar que el estudio se ha realizado en tres comunidades autónomas, Galicia, Valencia y Baleares y, dado que se centra en el ámbito recreativo, habría que considerar las diferencias geográficas y sociales a este respecto.

Futuras investigaciones deberían tratar de profundizar mediante métodos cualitativos en las dinámicas grupales de toma de decisión de los jóvenes sobre la asunción de conductas de riesgo en conducción.

\section{BIBLIOGRAFÍA}

1. Peden M, Scurfield R, Sleet D, Mohan D, Hyder AA, Jarawan E y Mathers C (eds). Informe mundial sobre prevención de los traumatismos causados por el tránsito Organización Mundial de la Salud. Washington: OMS; 2004.

2. Arranz JM, Gil AI, Accidentes de tráfico, víctimas mortales y consumo de alcohol. Madrid: Funda- 
ción de las cajas de ahorros; 2006. Documento de trabajo núm 262/2006.

3. Chipman ML, Macdonald S, Mann RE. Being "at fault" in traffic crashes: does alcohol, cannabis, cocaine, or polydrug abuse make a difference? Inj Prev. 2003 Dec;9(4):343-8.

4. Olivera C, Planes M, Conill M, Grass ME. Efectos del alcohol y conducción de vehículos: creencias y conductas de los jóvenes. Rev Esp Drogodep 2002; 27(1):66-80.

5. Allaert F, Aquatias S, Ballion R, Beauverie P, Cagni G, Bessin M, Colombie T, Delorme C, Dugarrin J, Emmanuelli J et al. Drugs and drug addictions. Indicators and Trends. Costes JM, editor. París: OFDT French Monitoring Centre for Drugs and Drug Adictions; 2005.

6. Ashton $\mathrm{CH}$. Pharmacology and effects of cannabis: a brief review. Br J Psychiatry. 2001; 2001(178):101-6.

7. Beirness DJ, Davis CG. Driving under the influence of Cannabis.Analisys Drawn from the 2004 Canadian Addiction Survey. Ottawa, ON: Canadian Center of Substance Abuse; 2006.

8. González Luque JC, Valdés E, Álvarez González FJ. Cannabis y conducción de vehículos: nuevas evidencias. Tráfico. 2004; 42.

9. Krüger HP, Vollrath M. Effects of cannabis and amphetamines on driving simulator performance of recreational drug users in the natural field. Center for Traffic Sciences (IZVW) 1998 [citado el 20 octubre 2007] Disponible en: http:// www.archido.de/indexeng.html

10. Willete. Drugs and Driving. Washinton: NIDA, department of health education and welfare. Washington: Public Health Service; 1977.

11. Sánchez Martín F. Actitudes sociales frente al riesgo vial en Europa. Resultados de la muestra española 2005. [citado el 20 de enero de 2008]. Disponible en: http://sartre.inrets.fr/english/sartre3E/Booklets/spanish/Page01.htm.

12. Bergdahl J, Norris MR. Sex differences in single vehicle fatal crashes: a research note. Soc Sci J. 2002; 39(2):287-293.
13. Keskinen E, Rajalin S. Comparison of young male and female drivers' attitude and self-reported traffic behaviour in Finland in 1978 and 2001. J Safety Res. 2003; 34(5): 579-87.

14. Calafat A, Fernández C, Juan M, Becoña E. Vida recreativa nocturna de los jóvenes españoles como factor de riesgo frente a otros más tradicionales. Adicciones 2006; 19(2):125-132.

15. Robert M. Good Practice in Managing the Evening and Late Night Economy: A Literature Review from an Environmental Perspective. London: Office of the Deputy Prime Minister; 2004.

16. Hadfield P. Bar Wars. Contesting the Night in Contemporary British Cities. Oxford: Oxford University Press; 2007.

17. Chatterton P, Hollands R. Urban nightscapes. Youth cultures, pleasure spaces and corporate power. Nueva York: Routledge; 2003.

18. Winlow S, Hall S. Violent night. Urban Leisure and Comtemporary Culture. Nueva York: Berg; 2006.

19. Degenhardt L, Dillon P, Duff C, Ross J. Driving, drug use behaviour and risk perceptions of nightclub attendees in Victoria, Australia. Int J Drug Policy. 2006; 17(1): 41-46.

20. Duff C, Rowland B. 'Rushing behind the wheel': Investigating the prevalence of 'drug driving' among club and rave patrons in Melbourne, Australia. Drugs: Education, Prevention \& Policy. 2006; 13(4): 299-312.

21. Jiang Y; Robin W S Drinking-Driving and Riding with Drunk Drivers among Young Adults: An Analysis of Reciprocal Effects. J Stud Alcohol. 1999; 60: 615-621.

22. Heckathorn, D. Respondent-Driven Sampling II: Deriving Valid Population Estimates from ChainReferral Samples of Hidden Populations". Soc Probl 2002; 49: 11-34.

23. Anderson P, Baumberg B. Report: alcohol in Europe. Conclusions and recommendations.. Brussels: European comisión; 2006, pp. 397-418. 
